were undertaken including staff education, updating of the referral form used by maternity staff and development of a neonatal antibiotic protocol. A re-audit was done in October 2014.

Results 18 babies were included in the initial audit and 19 babies on re-audit.

Vitamin K administration increased from 56\% to $100 \%$.

Antibiotics were given to $100 \%$ of babies in August 2014 as per hospital policy and to 95\% of babies in October 2014 following the introduction of a neonatal antibiotic protocol. Half of gentamicin doses (2/4) given to preterm babies were incorrect in Aug 2014 but all were correct (9/9) on re-audit. History of risk factors for sepsis was taken in $0 \%$ of admissions in August 2014. In October 2014 this increased to 16\%. The updated referral sheet which includes risk factors for infection is not yet widely used by maternity staff.

All observations were performed more frequently on re-audit, with respiratory rate recording improving from an average of 4.7 recordings in the first $24 \mathrm{~h}$ in August 2014 to 11.9 recordings in October 2014.

Recording of discharge weights in babies, who were discharged after 5 days or more, improved from 33\% to $92 \%$.

Conclusions Improvements in Vitamin $\mathrm{K}$ administration, neonatal observations, antibiotic prescribing and recording of discharge weights have been achieved through the audit process. Simple low cost interventions can result in better patient care in a low income setting.

There are challenges in implementing changes. Moving forward we hope to improve the history and documentation of risk factors for infection, by more consistent use of the updated referral sheet.

\section{G253 TRAINING LOCAL STAFF IN NORTHERN ETHIOPIA IN NEWBORN RESUSCITATION AND CARE}

V Hemming. General Paediatrics, Bradford Royal Infirmary, Bradford, UK

\subsection{6/archdischild-2015-308599.246}

There were 3.1 million neonatal deaths in 2010. The majority of these deaths occur in the developing world, particularly in SubSaharan Africa and South East Asia. Although the under fivechild mortality rate in Ethiopia from has improved from 204 to 68 per 1000 in $2012\left(156^{\text {th }}\right.$ worldwide) the neonatal mortality rate has been slow to improve. The neonatal mortality rate for Tigray region is high at 44 per 1000 compared to the national rate of 29 per 1000 (168 ${ }^{\text {th }}$ worldwide). The commonest causes of neonatal death are sepsis, birth asphyxia, and prematurity. Improvement in knowledge and skills associated with newborn care, early identification of problems and improvement in newborn resuscitation skills can improve outcomes.

Aim Develop a two-day training course for local hospital staff at a district hospital in the Northern Tigray region of Ethiopia on newborn resuscitation and essential newborn care and then train hospital staff.

Method A two-day training course was developed using country and regional guidelines and resuscitation standards. Teaching was delivered through lectures, small group work, skills teaching, and scenario practice. The course was delivered to four times in total to 83 hospital staff (doctors, nurses, clinical officers and midwives) overall. Pre and post course written tests and a post course practical test in newborn resuscitation were undertaking by all candidates.
Results There was a significant improvement between pre course and post course written test scores with a pre course average of $53.2 \%$ and a post course average of $66.4 \%(\mathrm{p}<0.001)$. The post course practical test score average was 5.5 out of 6 . Candidates provided positive feedback on the course.

Conclusion Training in newborn resuscitation and care is essential as part of improving neonatal morbidity and mortality. Local training programmes for hospital staff can improve knowledge and skills, which are then used in routine practice. It is important that these are accessible to all staff through using local languages to teach and training local staff as course faculty.

\section{G254(P) THE IMPACT OF AN OBSTETRIC BIRTH AND NEONATAL EMERGENCY LIFE SUPPORT EDUCATION PROJECT ON STAFF COMPETENCES, ORGANISATIONAL CAPACITY AND PATIENT OUTCOMES IN A RESOURCE - POOR SETTING}

JO Menakaya. Neonatal Paediatrics, Hillingdon Hospital, Uxbridge, UK

\subsection{6/archdischild-2015-308599.247}

Aims To design, deliver and evaluate the impact of a multi-structured educational programme on the management of obstetric and neonatal emergencies in a resource poor setting.

Methods I assessed the impact of a unique multi structured educational programme on Obstetric Birth and Neonatal Emergency Life Support delivered to health care workers in North Eastern Uganda using the Kirkpartrick model. Quantitative assessment methods included analysis of results of a questionnaire assessment of participants' pre and post course knowledge base and the number of early neonatal deaths pre and post course. T- test statistics was used to analyse the results. Qualitative assessment methods included structured and semi structured direct observation of skills and interviews with trained staff in the clinical settings 2 weeks and 6 months post course.

Results 138 health care workers participated in the training course delivered over two days for qualified staff and one day for students. $85 \%$ of participants completed a before and after multiple choice questionnaire evaluating knowledge of obstetric birth emergencies. Across the whole group, there was a statistically significant increase in post course scores by $10 \%$ compared with pre course scores. $97.8 \%$ of the qualified staff that underwent a practical assessment of skills at new born resuscitation were considered competent. On site evaluation of skill and practice 2 weeks post course revealed that most students used their new skills competently in a resuscitation scenario but highlighted a 'competency gap' in some cases. Evaluation of staff progress at 6 months revealed significant organisational challenges that affected staff training and development. Analysis of neonatal deaths showed that there was a $30 \%$ reduction in number of deaths post course compared with a similar time epoch two years earlier.

Conclusion This project demonstrated that UK style emergency life support courses on Obstetric Birth and Neonatal Life Emergencies can be delivered successfully in resource poor settings. The training led to newly acquired skill sets that in turn led to improvements in individual knowledge, organisational capabilities and neonatal mortality data. The challenge faced by organisers of educational programmes in similar settings is how to ensure that the new skills are embedded in order to sustain long term continuing improvements in clinical outcomes. 